careful monographs on particular cultures. The 'new anthropology' aims at discovering general laws, but it is doubtful whether such general laws, if discovered, would be of much service in investigating social phenomena.

On the other hand, the view expressed by Prof. Radcliffe-Brown that "no insight, however genial, can fully compensate for the absence of direct personal contact with the kind of material that the anthropologist has to study and explain", is to be fully endorsed. It is borne out by Prof. Westermarck's personal experience of nine years close contact with the Moors, spread over more than three decades, and spent in investigating their customs and ideas.

\title{
Morphogenesis and the Field Concept
}

\begin{abstract}
$\mathrm{T}$ HE discussion on morphogenesis which was held in Section D (Zoology) during the Blackpool meeting of the British Association was concerned mainly with the use of the field concept in experimental embryology. The central problem of the causal analysis of development is to account for the production of organs, that is to say, of masses of tissue which are integrated into units. Such integration can only be dealt with in terms of something which extends throughout the whole volume of the mass, and such extended entities are now usually referred to as 'fields'.
\end{abstract}

The first problem which arises is to give a satisfactory description of the integral behaviour. The sequence of shapes which are assumed by an organ during development has been described in great detail for most animal organs, but we require now some way of summing up, for each organ, the whole assemblage of shapes in a way which expresses the unitary character underlying the series. Probably a new branch of mathematics will be necessary to deal with such unfolding of even more complicated forms, though Dr. J. Needham mentioned the application of topological analysis and Gaussian curve analysis as hopeful possibilities. Mr. C. H. Waddington gave an example of what can already be attempted in some of the simpler cases; the shape of the notochord and somites of the early chick embryo can be expressed as the product of two damped wave functions, with the time variable in the expression for the damping of the longitudinal wave function, so that as time proceeds more waves (that is, somites) are formed. It is possible, by causing the embryonic body to develop in a bent form, to distort the co-ordinate network in which the functions are measured, and experiments of this kind may make it possible to choose, from mathematical possibilities, the particular formulation which most immediately expresses the actual forces at work.

The integrated or organized behaviour of developing organs is shown by the regulation of parts of the organ to reconstitute the whole; such experiments make it clear that the behaviour of a part of a developing organ is a function of its position in the whole. Dr. P. D. F. Murray deseribed some beautiful examples of this type of behaviour, taken from his own earlier work on chorio-allantoic grafts of fragments of the limb-bud of chick embryos, and from Miss H. B. Fell's recent work on tissue culture of the same material. Thus if the isolated fragment contains slightly more tissue than would normally take part in the formation of the femur, it never. theless forms no more than a complete femur, which therefore incorporates the extra tissue. Dr. Murray also discussed the mosaic development of later stages in which regulation is no longer possible. Prof. E. A.
Spaul's interesting and important communication on the hormonal control of metamorphosis in Amphibia also dealt with aspects of morphogenesis which do not come into the scope of this short note.

Regulation can only be analysed in ordinary causal terms if we suppose that the series of whole unit shapes, to which regulation occurs, is an equilibrium-series, that is, an equilibrium which is not constant but follows a definite course in time. According to this view, all the forces which at any time produce the developmental form-changes of an organ are in equilibrium with one another, and if the equilibrium is disturbed, for example, by removing part of the tissue, the same forces restore the equilibrium and thus cause the regulation of the organ. Regulation is therefore due to the interaction of forces proceeding from the different parts of the developing organ-rudiment. In so far as it is dependent on forces proceeding from different parts, it is dependent on local differences within the organ rudiment, and is in this respect similar to mosaic development, which is often and wrongly taken to be the exactly opposite type of behaviour to regulation. According to the present point of view, the differences between them are much less important; both types of behaviour depend upon local differences within the developing mass, but in cases where regulation occurs these differences are still labile and are capable of being altered by their mutual interaction, while in the mosaic stage the differences have become fixed and stable.

Dr. Needham concerned himself mainly with the nature of the forces which are concerned in setting up such equilibria. He directed particular attention to the possibility of finding a basis for the structural differentiation of masses of tissue in the arrangement of anisomorphic micelles or molecular aggregates. We know both that such molecules exist in protoplasm and that they can be arranged into orientated masses of the nature of liquid crystals. Such facts make it possible for the biochemist to form a picture of what it is the embryologist may be referring to when he invokes a 'cytosquellette' or an 'Intimstruktur'. The time has not yet come, he said, for an attempt at a detailed application of such ideas. We want to know, in particular, something about the maximum size of the volumes which can be affected by such molecular structures. Can we expect the orientated arrangements to be large enough to be invoked as an immediate explanation of such phenomena as the closure of the neural plate or the gastrulation movements in an amphibian egg, or must we only expect to find them active as deter. mining the positions of other agencies which are the immediate causes of the more large-scale changes of form?
C. H. WADDINGTON. 